\title{
Positive solutions of beam equations under nonlocal boundary value conditions
}

\author{
Shenglin Wang ${ }^{1}$, Jialong Chai ${ }^{1}$ and Guowei Zhang ${ }^{1 *}$ (i)
}

\begin{tabular}{l}
\hline${ }^{*}$ Correspondence: \\
gwzhang@mail.neu.edu.cn; \\
gwzhangneum@sina.com \\
'Department of Mathematics, \\
Northeastern University, Shenyang, \\
China
\end{tabular}

China

\begin{abstract}
In this article, we study the fourth-order problem with the first and second derivatives in nonlinearity under nonlocal boundary value conditions

$$
\begin{cases}u^{(4)}(t)=h(t) f\left(t, u(t), u^{\prime}(t),\right. & \left.u^{\prime \prime}(t)\right), \quad t \in(0,1), \\ u(0)=u(1)=\beta_{1}[u], & u^{\prime \prime}(0)+\beta_{2}[u]=0,\end{cases}
$$

where $f:[0,1] \times \mathbb{R}_{+} \times \mathbb{R} \times \mathbb{R}_{-} \rightarrow \mathbb{R}_{+}$is continuous, $h \in L^{1}(0,1)$ and $\beta_{i}[u]$ is Stieltjes integral $(i=1,2,3)$. This equation describes the deflection of an elastic beam. Some inequality conditions on nonlinearity $f$ are presented that guarantee the existence of positive solutions to the problem by the theory of fixed point index on a special cone in $C^{2}[0,1]$. Two examples are provided to support the main results under mixed boundary conditions involving multi-point with sign-changing coefficients and integral with sign-changing kernel.
\end{abstract}

MSC: Primary 34B18; 34B10; secondary 34B15

Keywords: Positive solution; Fixed point index; Cone

\section{Introduction}

In this article, we study the existence of positive solutions for fourth-order boundary value problem (BVP) with dependence on the first and second derivatives in nonlinearity subject to boundary conditions of Stieltjes integral type

$$
\left\{\begin{array}{l}
u^{(4)}(t)=h(t) f\left(t, u(t), u^{\prime}(t), u^{\prime \prime}(t)\right), \quad t \in(0,1), \\
u(0)=u(1)=\beta_{1}[u], \quad u^{\prime \prime}(0)+\beta_{2}[u]=0, \quad u^{\prime \prime}(1)+\beta_{3}[u]=0
\end{array}\right.
$$

where $\beta_{i}[u]=\int_{0}^{1} u(t) d \mathcal{B}_{i}(t)$ is Stieltjes integral with $\mathcal{B}_{i}$ of bounded variation $(i=1,2,3)$. This equation describes the deflection of an elastic beam.

Alves et al. [1] established the existence of positive solutions for the beam equation

$$
u^{(4)}(t)=f\left(t, u(t), u^{\prime}(t)\right)
$$

under boundary conditions

$$
u(0)=u^{\prime}(0)=0, \quad u^{\prime \prime \prime}(1)=g(u(1)), \quad u^{\prime}(1)=0 \quad \text { or } \quad u^{\prime \prime}(1)=0,
$$

(c) The Author(s) 2019. This article is distributed under the terms of the Creative Commons Attribution 4.0 International License (http://creativecommons.org/licenses/by/4.0/), which permits unrestricted use, distribution, and reproduction in any medium, provided you give appropriate credit to the original author(s) and the source, provide a link to the Creative Commons license, and indicate if changes were made. 
where $g$ is a continuous function. Using of the monotonically iterative technique, Yao [2] investigated the positive solution for fourth-order two-point boundary value problem

$$
\left\{\begin{array}{l}
u^{(4)}(t)=f\left(t, u(t), u^{\prime}(t)\right), \quad t \in(0,1) \\
u(0)=u^{\prime}(0)=u^{\prime \prime}(1)=u^{\prime \prime \prime}(1)=0
\end{array}\right.
$$

$\mathrm{Li}$ [3] and $\mathrm{Ma}$ [4] dealt with the existence of positive solutions for the fourth-order boundary value problem

$$
\left\{\begin{array}{l}
u^{(4)}(t)=f\left(t, u(t), u^{\prime \prime}(t)\right), \quad t \in(0,1) \\
u(0)=u^{\prime \prime}(0)=u(1)=u^{\prime \prime}(1)=0
\end{array}\right.
$$

Their methods are respectively based on fixed point index theory on cones and global bifurcation techniques. Bai [5] and Guo et al. [6] explored the existence of positive solutions respectively for the nonlocal fourth-order problems

$$
u^{(4)}(t)+\beta u^{\prime \prime}(t)=\lambda f\left(t, u(t), u^{\prime \prime}(t)\right)
$$

and

$$
u^{(4)}(t)+\beta u^{\prime \prime}(t)=\lambda f\left(t, u(t), u^{\prime}(t), u^{\prime \prime}(t), u^{\prime \prime \prime}(t)\right)
$$

subject to the same boundary conditions

$$
u(0)=u(1)=\int_{0}^{1} p(s) u(s) d s, \quad u^{\prime \prime}(0)=u^{\prime \prime}(1)=\int_{0}^{1} q(s) u^{\prime \prime}(s) d s,
$$

where $p, q \in L[0,1]$ are nonnegative. $\mathrm{Li}[7]$ discussed the existence of positive solutions for a local fully nonlinear problem

$$
\left\{\begin{array}{l}
u^{(4)}(t)=f\left(t, u(t), u^{\prime}(t), u^{\prime \prime}(t), u^{\prime \prime \prime}(t)\right), \quad t \in[0,1] \\
u(0)=u^{\prime}(0)=u^{\prime \prime}(1)=u^{\prime \prime \prime}(1)=0
\end{array}\right.
$$

where $f:[0,1] \times \mathbb{R}_{+}^{3} \times \mathbb{R}_{-} \rightarrow \mathbb{R}_{+}$is continuous. Under the conditions that the nonlinearity $f\left(t, x_{1}, x_{2}, x_{3}, x_{4}\right)$ may have superlinear or sublinear growth in $x_{1}, x_{2}, x_{3}, x_{4}$, the existence of positive solutions is obtained. We also refer to some previous studies, for instance, [8-12]. Recently the existence of positive solutions was proved in [13] to the following problems:

$$
\left\{\begin{array}{l}
u^{(4)}(t)=f\left(t, u(t), u^{\prime}(t), u^{\prime \prime}(t), u^{\prime \prime \prime}(t)\right), \quad t \in[0,1], \\
u^{\prime}(0)+\beta_{1}[u]=0, \quad u^{\prime \prime}(0)+\beta_{2}[u]=0, \quad u(1)=\beta_{3}[u], \quad u^{\prime \prime \prime}(1)=0,
\end{array}\right.
$$

and

$$
\left\{\begin{array}{l}
-u^{(4)}(t)=g\left(t, u(t), u^{\prime}(t), u^{\prime \prime}(t), u^{\prime \prime \prime}(t)\right), \quad t \in[0,1], \\
u(0)=\alpha_{1}[u], \quad u^{\prime}(0)=\alpha_{2}[u], \quad u^{\prime \prime}(0)=\alpha_{3}[u], \quad u^{\prime \prime \prime}(1)=0,
\end{array}\right.
$$

where $\beta_{i}[u]$ and $\alpha_{i}[u](i=1,2,3)$ are Stieltjes integrals of signed measures. All the signs of the derivatives from the first to the third with respect to $t$ of the Green's functions corresponding to (1.2) and (1.3) do not change, which plays an essential role in [13] when 
estimating the norms. The readers are referred to $[14,15]$ for more information and techniques about the issue considered.

Note that the boundary conditions in (1.1) are different from those in (1.2) and (1.3), and both the first and third derivatives with respect to $t$ of the Green's function corresponding to (1.1) may be sign-changing. We reformulate BVP (1.1) as an integral equation by the method due to Webb and Infante [16], see also $[17,18]$. If $u(0)=u(1)$, the existence of positive solutions to the resulting integral equation is tackled by the theory of fixed point index on a special cone in $C^{2}[0,1]$ under the inequality conditions posed on the nonlinearity. In particular, the fixed point indexes are computed via the cone expansion and compression conditions of functional type. Two examples are provided to support the main results under mixed boundary conditions involving multi-point with sign-changing coefficients and integral with sign-changing kernel.

\section{Preliminaries}

In order to prove the main theorems, we need the notion of a fixed point index; see, for example, [19,20]. Let $X$ be a Banach space, a nonempty subset $K$ is called a cone in $X$ if it is a closed convex set and satisfies the properties that $\lambda x \in K$ for any $\lambda>0, x \in K$, and that $\pm x \in K$ implies $x=0$ (the zero element in $X$ ). We say that $\alpha: K \rightarrow[0,+\infty)$ is a sublinear functional if $\alpha(t x) \leq t \alpha(x)$ for all $x \in K, t \in[0,1]$. The following lemmas come from [21].

Lemma 2.1 Let $K$ be a cone in Banach space $X$ and $\Omega$ be a bounded open subset relative to $K$ with $0 \in \Omega, S: \bar{\Omega} \rightarrow K$ is a completely continuous operator. Suppose that $\alpha: K \rightarrow$ $[0,+\infty)$ is a continuous and sublinear functional with $\alpha(0)=0, \alpha(x) \neq 0$ for $x \neq 0$. If $S x \neq x$ and $\alpha(S x) \leq \alpha(x)$ for all $x \in \partial \Omega$, then the fixed point index $i(S, \Omega, K)=1$.

Lemma 2.2 Let $K$ be a cone in Banach space $X$ and $\Omega$ be a bounded open subset relative to $K$ with $0 \in \Omega, S: \bar{\Omega} \rightarrow K$ is a completely continuous operator. Suppose that $\alpha: K \rightarrow[0,+\infty)$ is a continuous and sublinear functional with $\alpha(0)=0, \alpha(x) \neq 0$ for $x \neq 0$, and $\inf _{x \in \partial \Omega} \alpha(x)>0$. If $S x \neq x, \alpha(S x) \geq \alpha(x)$ for all $x \in \partial \Omega$, then the fixed point index $i(S, \Omega, K)=0$.

Let $X=C^{2}[0,1]$ be the Banach space consisting of all twice continuously differentiable functions on $[0,1]$ with the norm

$$
\|u\|_{C^{2}}=\max \left\{\|u\|_{C},\left\|u^{\prime}\right\|_{C^{\prime}},\left\|u^{\prime \prime}\right\|_{C}\right\}
$$

where $\|u\|_{C}=\max \{|u(t)|: t \in[0,1]\}$ for $u \in C[0,1]$. Define an operator in $C^{2}[0,1]$ as

$$
(T u)(t)=\sum_{i=1}^{3} \beta_{i}[u] \gamma_{i}(t)+\int_{0}^{1} k_{0}(t, s) h(s) f\left(s, u(s), u^{\prime}(s), u^{\prime \prime}(s)\right) d s,
$$

where $\gamma_{1}(t)=1, \gamma_{2}(t)=\frac{1}{6} t(1-t)(5-t), \gamma_{3}(t)=\frac{1}{6} t(1-t)(1+t)$,

$$
k_{0}(t, s)= \begin{cases}\frac{1}{6} t(1-s)\left(2 s-t^{2}-s^{2}\right), & 0 \leq t \leq s \leq 1, \\ \frac{1}{6} s(1-t)\left(2 t-s^{2}-t^{2}\right), & 0 \leq s \leq t \leq 1,\end{cases}
$$


in which $\beta_{i}[u]=\int_{0}^{1} u(t) d \mathcal{B}_{i}(t)(i=1,2,3)$. We set

$$
(B u)(t)=: \sum_{i=1}^{3} \beta_{i}[u] \gamma_{i}(t), \quad(F u)(t)=: \int_{0}^{1} k_{0}(t, s) h(s) f\left(s, u(s), u^{\prime}(s), u^{\prime \prime}(s)\right) d s,
$$

so $(T u)(t)=(B u)(t)+(F u)(t)$.

We assume throughout this paper that

$\left(C_{1}\right) f:[0,1] \times \mathbb{R}_{+} \times \mathbb{R} \times \mathbb{R}_{-} \rightarrow \mathbb{R}_{+}$is continuous; here $\mathbb{R}_{+}=[0, \infty)$ and $\mathbb{R}_{-}=(-\infty, 0]$, $h \in L^{1}(0,1)$ with $h(t) \geq 0$ and $\int_{0}^{1} h(t) d t>0$.

$\left(C_{2}\right)$ For each $i \in\{1,2,3\}, \mathcal{B}_{i}$ is of bounded variation and

$$
\mathcal{K}_{i}(s):=\int_{0}^{1} k_{0}(t, s) d \mathcal{B}_{i}(t) \geq 0, \quad \forall s \in[0,1]
$$

$\left(C_{3}\right) \beta_{i}\left[\gamma_{j}\right] \geq 0(i, j=1,2,3)$ and for the $3 \times 3$ matrix

$$
[B]=\left(\begin{array}{lll}
\beta_{1}\left[\gamma_{1}\right] & \beta_{1}\left[\gamma_{2}\right] & \beta_{1}\left[\gamma_{3}\right] \\
\beta_{2}\left[\gamma_{1}\right] & \beta_{2}\left[\gamma_{2}\right] & \beta_{2}\left[\gamma_{3}\right] \\
\beta_{3}\left[\gamma_{1}\right] & \beta_{3}\left[\gamma_{2}\right] & \beta_{3}\left[\gamma_{3}\right]
\end{array}\right)
$$

its spectral radius $r([B])<1$.

Writing $\langle\beta, \gamma\rangle=\sum_{i=1}^{3} \beta_{i} \gamma_{i}$ for the inner product in $\mathbb{R}^{3}$, we define the operator $S$ in $C^{2}[0,1]$ as

$$
(S u)(t)=\left\langle(I-[B])^{-1} \beta[F u], \gamma(t)\right\rangle+(F u)(t),
$$

where $\beta[F u]=\left(\beta_{1}[F u], \beta_{2}[F u], \beta_{3}[F u]\right)^{T}$ is the transposed vector. Similar to [16] we have the following lemmas.

Lemma 2.3 Suppose that $\left(C_{1}\right)$ holds. Then BVP (1.1) has a solution if and only if there exists a fixed point of $T$ in $C^{2}[0,1]$.

Lemma 2.4 Suppose that $\left(C_{1}\right)-\left(C_{3}\right)$ hold. Then $S$ can be written as

$$
\begin{aligned}
(S u)(t) & =\left((I-B)^{-1} F u\right)(t) \\
& =\int_{0}^{1}\left(\left\langle(I-[B])^{-1} \mathcal{K}(s), \gamma(t)\right\rangle+k_{0}(t, s)\right) h(s) f\left(s, u(s), u^{\prime}(s), u^{\prime \prime}(s)\right) d s \\
& =: \int_{0}^{1} k_{S}(t, s) h(s) f\left(s, u(s), u^{\prime}(s), u^{\prime \prime}(s)\right) d s,
\end{aligned}
$$

where $\mathcal{K}(s)=\left(\mathcal{K}_{1}(s), \mathcal{K}_{2}(s), \mathcal{K}_{2}(s)\right)^{T}$, i.e.,

$$
k_{S}(t, s)=\left\langle(I-[B])^{-1} \mathcal{K}(s), \gamma(t)\right\rangle+k_{0}(t, s)=\sum_{i=1}^{3} \kappa_{i}(s) \gamma_{i}(t)+k_{0}(t, s)
$$

and $\kappa_{i}(s)$ is the ith component of $(I-[B])^{-1} \mathcal{K}(s)$. 
Lemma 2.5 If $\left(C_{2}\right)$ and $\left(C_{3}\right)$ hold, then $\kappa_{i}(s) \geq 0(i=1,2)$,

$$
k_{S}(0, s)=k_{S}(1, s)=\kappa_{1}(s)
$$

and for $t, s \in[0,1]$,

$$
c_{0}(t) \Phi_{0}(s) \leq k_{S}(t, s) \leq \Phi_{0}(s)
$$

where

$$
\begin{aligned}
& \Phi_{0}(s)=\sum_{i=1}^{3} \kappa_{i}(s)+\widetilde{\Phi}_{0}(s), \quad c_{0}(t)=\widetilde{c}_{0}(t)+\gamma_{3}(t), \\
& \tilde{c}_{0}(t)= \begin{cases}\frac{3 \sqrt{3}}{2} t\left(1-t^{2}\right), & 0 \leq t \leq \frac{1}{2}, \\
\frac{3 \sqrt{3}}{2} t(1-t)(2-t), & \frac{1}{2}<t \leq 1,\end{cases} \\
& \widetilde{\Phi}_{0}(s)= \begin{cases}\frac{\sqrt{3}}{27} s\left(1-s^{2}\right)^{3 / 2}, & 0 \leq s \leq \frac{1}{2}, \\
\frac{\sqrt{3}}{27}(1-s) s^{3 / 2}(2-s)^{3 / 2}, & \frac{1}{2}<s \leq 1 ;\end{cases}
\end{aligned}
$$

and

$$
c_{1}(t) \Phi_{1}(s) \leq-\frac{\partial^{2} k_{S}(t, s)}{\partial t^{2}} \leq \Phi_{1}(s)
$$

where $\Phi_{1}(s)=2 \kappa_{2}(s)+\kappa_{3}(s)+s(1-s), c_{1}(t)=\min \{t,(1-t) / 2\}$.

Proof Inequality $\kappa_{i}(s) \geq 0$ is due to [16] and we can find in [18] the inequalities

$$
\widetilde{c}_{0}(t) \widetilde{\Phi}_{0}(s) \leq k_{0}(t, s) \leq \widetilde{\Phi}_{0}(s) .
$$

As for (2.7), it can be checked easily.

Define a cone $K$ in $C^{2}[0,1]$ as follows:

$$
\begin{aligned}
P= & \left\{u \in C^{2}[0,1]: u(t) \geq 0, u^{\prime \prime}(t) \leq 0, \forall t \in[0,1]\right\}, \\
K= & \left\{u \in P: u(0)=u(1), u(t) \geq c_{0}(t)\|u\|_{C},\right. \\
& \left.-u^{\prime \prime}(t) \geq c_{1}(t)\left\|u^{\prime \prime}\right\|_{C}, \forall t \in[0,1] ; \beta_{i}[u] \geq 0(i=1,2,3)\right\} .
\end{aligned}
$$

Lemma 2.6 If $\left(C_{1}\right)-\left(C_{3}\right)$ hold, then $S: P \rightarrow K$ is a completely continuous operator.

Proof For $u \in P$ and $t \in[0,1]$, it is easy to see that $S u \in C^{2}[0,1],(S u)(t) \geq 0$ and $(\mathrm{Su})^{\prime \prime}(t) \leq 0$. By Lemma 2.5,

$$
\begin{aligned}
(S u)(0) & =\int_{0}^{1} k_{S}(0, s) h(s) f\left(s, u(s), u^{\prime}(s), u^{\prime \prime}(s)\right) d s \\
& =\int_{0}^{1} k_{S}(1, s) h(s) f\left(s, u(s), u^{\prime}(s), u^{\prime \prime}(s)\right) d s=(S u)(1) .
\end{aligned}
$$


Also by Lemma 2.5,

$$
\begin{aligned}
(S u)(t) & =\int_{0}^{1} k_{S}(t, s) h(s) f\left(s, u(s), u^{\prime}(s), u^{\prime \prime}(s)\right) d s \\
& \geq c_{0}(t) \int_{0}^{1} \Phi_{0}(s) h(s) f\left(s, u(s), u^{\prime}(s), u^{\prime \prime}(s)\right) d s
\end{aligned}
$$

and

$$
\begin{aligned}
-(S u)^{\prime \prime}(t) & =-\int_{0}^{1} \frac{\partial^{2} k_{S}(t, s)}{\partial t^{2}} h(s) f\left(s, u(s), u^{\prime}(s), u^{\prime \prime}(s)\right) d s \\
& \geq c_{1}(t) \int_{0}^{1} \Phi_{1}(s) h(s) f\left(s, u(s), u^{\prime}(s), u^{\prime \prime}(s)\right) d s,
\end{aligned}
$$

hence we have

$$
\begin{aligned}
(S u)(t) & =\int_{0}^{1} k_{S}(t, s) h(s) f\left(s, u(s), u^{\prime}(s), u^{\prime \prime}(s)\right) d s \\
& \leq \int_{0}^{1} \Phi_{0}(s) h(s) f\left(s, u(s), u^{\prime}(s), u^{\prime \prime}(s)\right) d s
\end{aligned}
$$

and

$$
\begin{aligned}
-(S u)^{\prime \prime}(t) & =-\int_{0}^{1} \frac{\partial^{2} k_{S}(t, s)}{\partial t^{2}} h(s) f\left(s, u(s), u^{\prime}(s), u^{\prime \prime}(s)\right) d s \\
& \leq \int_{0}^{1} \Phi_{1}(s) h(s) f\left(s, u(s), u^{\prime}(s), u^{\prime \prime}(s)\right) d s,
\end{aligned}
$$

therefore $(S u)(t) \geq c_{0}(t)\|S u\|_{C}$ and $-(S u)^{\prime \prime}(t) \geq c_{1}(t)\left\|(S u)^{\prime \prime}\right\|_{C}$ for $t \in[0,1]$. Moreover, it follows from $\left(C_{2}\right)$ that

$$
\begin{aligned}
\beta_{i}[S u] & =\int_{0}^{1}\left(\int_{0}^{1} k_{S}(t, s) h(s) f\left(s, u(s), u^{\prime}(s), u^{\prime \prime}(s)\right) d s\right) d \mathcal{B}_{i}(t) \\
& =\int_{0}^{1}\left(\int_{0}^{1} k_{S}(t, s) d \mathcal{B}_{i}(t)\right) h(s) f\left(s, u(s), u^{\prime}(s), u^{\prime \prime}(s)\right) d s \\
& =\int_{0}^{1} \mathcal{K}_{i}(s) h(s) f\left(s, u(s), u^{\prime}(s), u^{\prime \prime}(s)\right) \geq 0 \quad(i=1,2,3),
\end{aligned}
$$

that is, $S u \in K$. The complete continuity of $S$ is obvious.

Lemma 2.7 If $\left(C_{1}\right)-\left(C_{3}\right)$ hold, then $S$ and $T$ have the same fixed points in $K$. As a result, $B V P(1.1)$ has a positive solution if and only if $S$ has a fixed point in $K$.

\section{Positive solutions of BVP}

Take $\tau \in(0,1 / 3)$ such that $\int_{\tau}^{1-\tau} h(t) d t>0$ and denote

$$
h_{0}=\max \left\{\int_{0}^{1} \Phi_{0}(t) h(t) d t, \int_{0}^{1} \Phi_{1}(t) h(t) d t\right\}
$$




$$
h_{\tau}=\min \left\{\int_{\tau}^{1-\tau} \Phi_{0}(t) h(t) d t, \int_{\tau}^{1-\tau} \Phi_{1}(t) h(t) d t\right\} .
$$

Define a functional $\alpha: K \rightarrow[0,+\infty)$ as

$$
\alpha(u)=\max \left\{\max _{\tau \leq t \leq 1-\tau}|u(t)|, \max _{\tau \leq t \leq 1-\tau}\left|u^{\prime \prime}(t)\right|\right\} .
$$

Clearly, $\alpha$ is a continuous and sublinear functional with $\alpha(0)=0$. Moreover, since

$$
\alpha(u) \geq \max _{\tau \leq t \leq 1-\tau}|u(t)| \geq\left(\max _{\tau \leq t \leq 1-\tau} c_{0}(t)\right)\|u\|_{C} \geq \frac{1}{16}(9 \sqrt{3}+1)\|u\|_{C}, \quad u \in K,
$$

it is easy to see that $\alpha(u) \neq 0$ for $u \neq 0$.

Theorem 3.1 Suppose that $\left(C_{1}\right)-\left(C_{3}\right)$ are satisfied. If there exist constants $a$ and $b$ with $0<b<a$ satisfying $3 b<\tau a$,

$$
f\left(t, x_{1}, x_{2}, x_{3}\right) \leq h_{0}^{-1} b
$$

for $\left(t, x_{1}, x_{2}, x_{3}\right) \in D_{1}=[0,1] \times[0,3 b] \times[-3 b, 3 b] \times[-3 b, 0]$ and

$$
f\left(t, x_{1}, x_{2}, x_{3}\right) \geq 3 h_{\tau}^{-1} a
$$

for $\left(t, x_{1}, x_{2}, x_{3}\right) \in D_{2} \cup D_{3}$, where

$$
\begin{aligned}
& D_{2}=[0,1] \times[\tau a, a] \times[-3 a, 3 a] \times[-3 a, 0], \\
& D_{3}=[0,1] \times[0,3 a] \times[-3 a, 3 a] \times[-a,-\tau a],
\end{aligned}
$$

then BVP (1.1) has at least one positive solution.

Proof Obviously, $D_{1} \cap\left(D_{2} \cup D_{3}\right)=\emptyset$ since $3 b<\tau a$. Let

$$
\Omega_{1}=\{u \in K: \alpha(u)<b\}, \quad \Omega_{2}=\{u \in K: \alpha(u)<a\},
$$

then it is clear that $\Omega_{1}$ and $\Omega_{2}$ are open sets in $K$ with $0 \in \Omega_{1}$ and $\bar{\Omega}_{1} \subset \Omega_{2}$.

If $u \in \Omega_{2}$, by Lemma 2.5 , we have

$$
a>\max _{\tau \leq t \leq 1-\tau}|u(t)| \geq\left(\max _{\tau \leq t \leq 1-\tau} c_{0}(t)\right)\|u\|_{C} \geq \frac{1}{16}(9 \sqrt{3}+1)\|u\|_{C} \geq \frac{1}{3}\|u\|_{C}
$$

and

$$
a>\max _{\tau \leq t \leq 1-\tau}\left|u^{\prime \prime}(t)\right| \geq\left(\max _{\tau \leq t \leq 1-\tau} c_{1}(t)\right)\left\|u^{\prime \prime}\right\|_{C}=\frac{1}{3}\left\|u^{\prime \prime}\right\|_{C} .
$$

Since $u(0)=u(1)$, there exists $\xi \in(0,1)$ such that $u^{\prime}(\xi)=0$ and thus

$$
\left\|u^{\prime}\right\|_{C}=\max _{0 \leq t \leq 1}\left|u^{\prime}(t)\right| \leq \max _{0 \leq t \leq 1}\left|\int_{\xi}^{t}\right| u^{\prime \prime}(s)|d s| \leq\left\|u^{\prime \prime}\right\|_{C}<3 a .
$$


Therefore, $\Omega_{2}$ is bounded and $\|u\|_{C^{2}}<3 a, \forall u \in \Omega_{2}$. Similarly, $\Omega_{1}$ is bounded and $\|u\|_{C^{2}}<$ $3 b, \forall u \in \Omega_{1}$.

If $u \in \partial \Omega_{1}$, then $\alpha(u)=b$ and $\|u\|_{C^{2}} \leq 3 b$. From Lemma 2.5 and (3.1) it follows that

$$
\begin{aligned}
\max _{\tau \leq t \leq 1-\tau}|(S u)(t)| & =\max _{\tau \leq t \leq 1-\tau}\left|\int_{0}^{1} k_{S}(t, s) h(s) f\left(s, u(s), u^{\prime}(s), u^{\prime \prime}(s)\right) d s\right| \\
& \leq h_{0}^{-1} b \int_{0}^{1} \Phi_{0}(s) h(s) d s \leq b, \\
\max _{\tau \leq t \leq 1-\tau}\left|(S u)^{\prime \prime}(t)\right| & =\max _{\tau \leq t \leq 1-\tau}\left|\int_{0}^{1} \frac{\partial^{2} k_{S}(t, s)}{\partial t^{2}} h(s) f\left(s, u(s), u^{\prime}(s), u^{\prime \prime}(s)\right) d s\right| \\
& \leq h_{0}^{-1} b \int_{0}^{1} \Phi_{1}(s) h(s) d s \leq b,
\end{aligned}
$$

and hence $\alpha(\mathrm{Su}) \leq \alpha(u)$. So by Lemma 2.1 the fixed point index

$$
i\left(S, \Omega_{1}, K\right)=1,
$$

provided $S u \neq u$ for $u \in \partial \Omega_{1}$.

If $u \in \partial \Omega_{2}$, then $\alpha(u)=a$ and, by Lemma 2.5 for $t \in[\tau, 1-\tau]$,

$$
\begin{aligned}
a & \geq u(t) \geq c_{0}(t)\|u\|_{C} \geq\left(\min _{\tau \leq t \leq 1-\tau} c_{0}(t)\right)\|u\|_{C} \\
& \geq\left(\frac{3 \sqrt{3}}{2}+\frac{1}{6}\right) \tau\left(1-\tau^{2}\right)\|u\|_{C} \geq \tau\|u\|_{C} \geq \tau \max _{\tau \leq t \leq 1-\tau}|u(t)|
\end{aligned}
$$

and

$$
\begin{aligned}
a & \geq-u^{\prime \prime}(t) \geq c_{1}(t)\left\|u^{\prime \prime}\right\|_{C} \geq\left(\min _{\tau \leq t \leq 1-\tau} c_{1}(t)\right)\|u\|_{C} \\
& =\tau\left\|u^{\prime \prime}\right\|_{C} \geq \tau \max _{\tau \leq t \leq 1-\tau}\left|u^{\prime \prime}(t)\right| .
\end{aligned}
$$

When $\alpha(u)=a=\max _{\tau \leq t \leq 1-\tau}|u(t)|$, it follows from Lemma 2.5, together with (3.2) and (3.4), that

$$
\begin{aligned}
\max _{\tau \leq t \leq 1-\tau}|(S u)(t)| & =\max _{\tau \leq t \leq 1-\tau}\left|\int_{0}^{1} k_{S}(t, s) h(s) f\left(s, u(s), u^{\prime}(s), u^{\prime \prime}(s)\right) d s\right| \\
& \geq\left(\max _{\tau \leq t \leq 1-\tau} c_{0}(t)\right) \int_{\tau}^{1-\tau} \Phi_{0}(s) h(s) f\left(s, u(s), u^{\prime}(s), u^{\prime \prime}(s)\right) d s \\
& \geq \frac{1}{3} 3 h_{\tau}^{-1} a \int_{\tau}^{1-\tau} \Phi_{0}(s) h(s) d s \geq a, \\
\max _{\tau \leq t \leq 1-\tau}\left|(S u)^{\prime \prime}(t)\right| & =\max _{\tau \leq t \leq 1-\tau}\left|\int_{0}^{1} \frac{\partial^{2} k_{S}(t, s)}{\partial t^{2}} h(s) f\left(s, u(s), u^{\prime}(s), u^{\prime \prime}(s)\right) d s\right| \\
& \geq\left(\max _{\tau \leq t \leq 1-\tau} c_{1}(t)\right) \int_{\tau}^{1-\tau} \Phi_{1}(s) h(s) f\left(s, u(s), u^{\prime}(s), u^{\prime \prime}(s)\right) d s \\
& \geq \frac{1}{3} 3 h_{\tau}^{-1} a \int_{\tau}^{1-\tau} \Phi_{1}(s) h(s) d s \geq a,
\end{aligned}
$$


and hence $\alpha(\mathrm{Su}) \geq \alpha(u)$; when $\alpha(u)=a=\max _{\tau \leq t \leq 1-\tau}\left|u^{\prime \prime}(t)\right|$, it similarly follows from Lemma 2.5, together with (3.2) and (3.5), that $\alpha(S u) \geq \alpha(u)$. So by Lemma 2.2 and since $\inf _{x \in \partial \Omega_{2}} \alpha(u)=a>0$, the fixed point index

$$
i\left(S, \Omega_{2}, K\right)=0,
$$

provided $S u \neq u$ for $u \in \partial \Omega_{2}$.

From (3.3) and (3.6) it follows that the fixed point index

$$
i\left(S, \Omega_{2} \backslash \bar{\Omega}_{1}, K\right)=i\left(S, \Omega_{2}, K\right)-i\left(S, \Omega_{1}, K\right)=-1,
$$

hence $S$ has at least one fixed solution and BVP (1.1) has at least one positive solution.

Theorem 3.2 Suppose that $\left(C_{1}\right)-\left(C_{3}\right)$ are satisfied. If there exist constants $a$ and $b$ with $0<b<a$ satisfying $a>3 h_{0} h_{\tau}^{-1} b$,

$$
f\left(t, x_{1}, x_{2}, x_{3}\right) \geq 3 h_{\tau}^{-1} b
$$

for $\left(t, x_{1}, x_{2}, x_{3}\right) \in D_{1} \cup D_{2}$, where

$$
\begin{aligned}
& D_{1}=[0,1] \times[\tau b, b] \times[-3 b, 3 b] \times[-3 b, 0], \\
& D_{2}=[0,1] \times[0,3 b] \times[-3 b, 3 b] \times[-b,-\tau b],
\end{aligned}
$$

and

$$
f\left(t, x_{1}, x_{2}, x_{3}\right) \leq h_{0}^{-1} a
$$

for $\left(t, x_{1}, x_{2}, x_{3}\right) \in D_{3}=[0,1] \times[0,3 a] \times[-3 a, 3 a] \times[-3 a, 0]$, then $B V P(1.1)$ has at least one positive solution.

Proof Obviously, $D_{1} \cup D_{2} \subset D_{3}$; however, (3.7) and (3.8) are well-posed since $a>3 h_{0} h_{\tau}^{-1} b$. Letting

$$
\Omega_{1}=\{u \in K: \alpha(u)<b\}, \quad \Omega_{2}=\{u \in K: \alpha(u)<a\},
$$

we know form the proof of Theorem 3.1 that $\Omega_{1}$ and $\Omega_{2}$ are bounded open sets in $K$ with $0 \in \Omega_{1}$ and $\bar{\Omega}_{1} \subset \Omega_{2}$; moreover, $\|u\|_{C^{2}}<3 b$ for $u \in \Omega_{1}$ and $\|u\|_{C^{2}}<3 a$ for $u \in \Omega_{2}$.

If $u \in \partial \Omega_{1}$, then $\alpha(u)=b$ and, by Lemma 2.5 for $t \in[\tau, 1-\tau]$,

$$
\begin{aligned}
b & \geq u(t) \geq c_{0}(t)\|u\|_{C} \geq\left(\min _{\tau \leq t \leq 1-\tau} c_{0}(t)\right)\|u\|_{C} \\
& \geq\left(\frac{3 \sqrt{3}}{2}+\frac{1}{6}\right) \tau\left(1-\tau^{2}\right)\|u\|_{C} \geq \tau\|u\|_{C} \geq \tau \max _{\tau \leq t \leq 1-\tau}|u(t)|
\end{aligned}
$$

and

$$
\begin{aligned}
b & \geq-u^{\prime \prime}(t) \geq c_{1}(t)\left\|u^{\prime \prime}\right\|_{C} \geq\left(\min _{\tau \leq t \leq 1-\tau} c_{1}(t)\right)\|u\|_{C} \\
& =\tau\left\|u^{\prime \prime}\right\|_{C} \geq \tau \max _{\tau \leq t \leq 1-\tau}\left|u^{\prime \prime}(t)\right| .
\end{aligned}
$$


When $\alpha(u)=b=\max _{\tau \leq t \leq 1-\tau}|u(t)|$, it follows from Lemma 2.5, as well as (3.7) and (3.9), that

$$
\begin{aligned}
\max _{\tau \leq t \leq 1-\tau}|(S u)(t)| & =\max _{\tau \leq t \leq 1-\tau}\left|\int_{0}^{1} k_{S}(t, s) h(s) f\left(s, u(s), u^{\prime}(s), u^{\prime \prime}(s)\right) d s\right| \\
& \geq\left(\max _{\tau \leq t \leq 1-\tau} c_{0}(t)\right) \int_{\tau}^{1-\tau} \Phi_{0}(s) h(s) f\left(s, u(s), u^{\prime}(s), u^{\prime \prime}(s)\right) d s \\
& \geq \frac{1}{3} 3 h_{\tau}^{-1} b \int_{\tau}^{1-\tau} \Phi_{0}(s) h(s) d s \geq b, \\
\max _{\tau \leq t \leq 1-\tau}\left|(S u)^{\prime \prime}(t)\right| & =\max _{\tau \leq t \leq 1-\tau}\left|\int_{0}^{1} \frac{\partial^{2} k_{S}(t, s)}{\partial t^{2}} h(s) f\left(s, u(s), u^{\prime}(s), u^{\prime \prime}(s)\right) d s\right| \\
& \geq\left(\max _{\tau \leq t \leq 1-\tau} c_{1}(t)\right) \int_{\tau}^{1-\tau} \Phi_{1}(s) h(s) f\left(s, u(s), u^{\prime}(s), u^{\prime \prime}(s)\right) d s \\
& \geq \frac{1}{3} 3 h_{\tau}^{-1} b \int_{\tau}^{1-\tau} \Phi_{1}(s) h(s) d s \geq b,
\end{aligned}
$$

and hence $\alpha(\mathrm{Su}) \geq \alpha(u)$; when $\alpha(u)=b=\max _{\tau \leq t \leq 1-\tau}\left|u^{\prime \prime}(t)\right|$, it similarly follows from Lemma 2.5, together with (3.7) and (3.10), that $\alpha(S u) \geq \alpha(u)$. So by Lemma 2.2 and since $\inf _{x \in \partial \Omega_{1}} \alpha(u)=b>0$, the fixed point index

$$
i\left(S, \Omega_{1}, K\right)=0,
$$

provided $S u \neq u$ for $u \in \partial \Omega_{1}$.

If $u \in \partial \Omega_{2}$, then $\alpha(u)=a$ and $\|u\|_{C^{2}} \leq 3 a$. From Lemma 2.5 and (3.8) it follows that

$$
\begin{aligned}
\max _{\tau \leq t \leq 1-\tau}|(S u)(t)| & =\max _{\tau \leq t \leq 1-\tau}\left|\int_{0}^{1} k_{S}(t, s) h(s) f\left(s, u(s), u^{\prime}(s), u^{\prime \prime}(s)\right) d s\right| \\
& \leq h_{0}^{-1} a \int_{0}^{1} \Phi_{0}(s) h(s) d s \leq a, \\
\max _{\tau \leq t \leq 1-\tau}\left|(S u)^{\prime \prime}(t)\right| & =\max _{\tau \leq t \leq 1-\tau}\left|\int_{0}^{1} \frac{\partial^{2} k_{s}(t, s)}{\partial t^{2}} h(s) f\left(s, u(s), u^{\prime}(s), u^{\prime \prime}(s)\right) d s\right| \\
& \leq h_{0}^{-1} a \int_{0}^{1} \Phi_{1}(s) h(s) d s \leq a,
\end{aligned}
$$

and hence $\alpha(S u) \leq \alpha(u)$. So by Lemma 2.1 the fixed point index

$$
i\left(S, \Omega_{2}, K\right)=1,
$$

provided $S u \neq u$ for $u \in \partial \Omega_{2}$.

From (3.11) and (3.12) it follows that the fixed point index

$$
i\left(S, \Omega_{2} \backslash \bar{\Omega}_{1}, K\right)=i\left(S, \Omega_{2}, K\right)-i\left(S, \Omega_{1}, K\right)=1,
$$

hence $S$ has at least one fixed solution and BVP (1.1) has at least one positive solution. 


\section{Examples}

We consider fourth-order problems under mixed boundary conditions involving multipoint with sign-changing coefficients and integral with sign-changing kernel

$$
\left\{\begin{array}{l}
u^{(4)}(t)=\frac{1}{\sqrt{t(1-t)}} f\left(t, u(t), u^{\prime}(t), u^{\prime \prime}(t)\right), \quad t \in(0,1), \\
u(0)=u(1)=\frac{1}{4} u\left(\frac{1}{4}\right)-\frac{1}{12} u\left(\frac{3}{4}\right), \\
u^{\prime \prime}(0)+\int_{0}^{1} u(t)\left(2 t-\frac{1}{2}\right) d t=0, \quad u^{\prime \prime}(1)+\frac{1}{2} u\left(\frac{1}{2}\right)-\frac{1}{4} u\left(\frac{3}{4}\right)=0,
\end{array}\right.
$$

that is, $\beta_{1}[u]=\frac{1}{4} u\left(\frac{1}{4}\right)-\frac{1}{12} u\left(\frac{3}{4}\right), \beta_{2}[u]=\int_{0}^{1} u(t)\left(2 t-\frac{1}{2}\right) d t, \beta_{3}[u]=\frac{1}{2} u\left(\frac{1}{2}\right)-\frac{1}{4} u\left(\frac{3}{4}\right)$. Hence for $s \in[0,1]$,

$$
\begin{aligned}
& 0 \leq \mathcal{K}_{1}(s)=\frac{1}{4} k_{0}\left(\frac{1}{4}, s\right)-\frac{1}{12} k_{0}\left(\frac{3}{4}, s\right) \\
&= \begin{cases}-\frac{1}{36} s^{3}+\frac{1}{96} s, & 0 \leq s \leq \frac{1}{4}, \\
\frac{1}{72} s^{3}-\frac{1}{32} s^{2}+\frac{7}{384} s-\frac{1}{1536}, & \frac{1}{4}<s \leq \frac{3}{4}, \\
-\frac{1}{192} s+\frac{1}{192}, & \frac{3}{4}<s \leq 1,\end{cases} \\
& \mathcal{K}_{2}(s)=\int_{0}^{1} k_{0}(t, s)\left(2 t-\frac{1}{2}\right) d t=\frac{1}{60} s^{5}-\frac{1}{48} s^{4}-\frac{1}{72} s^{3}+\frac{13}{720} s \geq 0, \\
& 0 \leq \mathcal{K}_{3}(s)=\frac{1}{2} k_{0}\left(\frac{1}{2}, s\right)-\frac{1}{4} k_{0}\left(\frac{3}{4}, s\right) \\
&= \begin{cases}-\frac{1}{32} s^{3}+\frac{11}{512} s, & 0 \leq s \leq \frac{1}{2}, \\
\frac{5}{96} s^{3}-\frac{1}{8} s^{2}+\frac{43}{512} s-\frac{1}{96}, & \frac{1}{2}<s \leq \frac{3}{4}, \\
\frac{1}{96} s^{3}-\frac{1}{32} s^{2}+\frac{7}{512} s+\frac{11}{1536}, & \frac{3}{4}<s \leq 1,\end{cases}
\end{aligned}
$$

and the $3 \times 3$ matrix

$$
[B]=\left(\begin{array}{lll}
\beta_{1}\left[\gamma_{1}\right] & \beta_{1}\left[\gamma_{2}\right] & \beta_{1}\left[\gamma_{3}\right] \\
\beta_{2}\left[\gamma_{1}\right] & \beta_{2}\left[\gamma_{2}\right] & \beta_{2}\left[\gamma_{3}\right] \\
\beta_{3}\left[\gamma_{1}\right] & \beta_{3}\left[\gamma_{2}\right] & \beta_{3}\left[\gamma_{3}\right]
\end{array}\right)=\left(\begin{array}{ccc}
\frac{1}{6} & \frac{5}{192} & \frac{1}{192} \\
0 & \frac{43}{720} & \frac{17}{720} \\
\frac{1}{4} & \frac{31}{512} & \frac{9}{512}
\end{array}\right)
$$

Its spectral radius is $r([B]) \approx 0.1832<1$. This means that $\left(C_{2}\right)$ and $\left(C_{3}\right)$ are satisfied. Moreover,

$$
\begin{aligned}
& \kappa_{1}(s)=1.2022 \mathcal{K}_{1}(s)+0.0338 \mathcal{K}_{2}(s)+0.0072 \mathcal{K}_{3}(s), \\
& \kappa_{2}(s)=0.0077 \mathcal{K}_{1}(s)+1.0654 \mathcal{K}_{2}(s)+0.0256 \mathcal{K}_{3}(s), \\
& \kappa_{3}(s)=0.3064 \mathcal{K}_{1}(s)+0.0742 \mathcal{K}_{2}(s)+1.0213 \mathcal{K}_{3}(s) .
\end{aligned}
$$

Take $\tau=1 / 4$ and then

$$
\begin{aligned}
& h_{0}=\max \left\{\int_{0}^{1} \Phi_{0}(t) h(t) d t, \int_{0}^{1} \Phi_{1}(t) h(t) d t\right\}=\max \{0.0578,0.4257\}=0.4257 \\
& h_{\tau}=\min \left\{\int_{1 / 4}^{3 / 4} \Phi_{0}(t) h(t) d t, \int_{1 / 4}^{3 / 4} \Phi_{1}(t) h(t) d t\right\}=\min \{0.0366,0.2600\}=0.0366
\end{aligned}
$$

Example 4.1 If $f\left(t, x_{1}, x_{2}, x_{3}\right)=x_{1}^{2}+\frac{1+t}{2} x_{2}^{2}+x_{3}^{2}$, then BVP (4.1) has a positive solution. 
Proof For $a=1600, b=0.01$, it is clear that $3 b<a / 4$. Moreover,

$$
f\left(t, x_{1}, x_{2}, x_{3}\right) \leq 3 \times\left(\frac{3}{100}\right)^{2}=0.0027<h_{0}^{-1} b=0.0235
$$

for $\left(t, x_{1}, x_{2}, x_{3}\right) \in D_{1}=[0,1] \times[0,0.03] \times[-0.03,0.03] \times[-0.03,0]$, and

$$
f\left(t, x_{1}, x_{2}, x_{3}\right) \geq 400^{2}>3 h_{\tau}^{-1} a
$$

for $\left(t, x_{1}, x_{2}, x_{3}\right) \in([0,1] \times[400,1600] \times[-4800,4800] \times[-4800,0]) \cup([0,1] \times[0,4800] \times$ $[-4800,4800] \times[-1600,-400])$. Then BVP $(4.1)$ has a positive solution by Theorem 3.1.

Example 4.2 If $f\left(t, x_{1}, x_{2}, x_{3}\right)=2000\left(1-\frac{1}{1+x_{1}^{2}+(1+t) x_{2}^{4}+x_{3}^{2}}\right)$, then BVP (4.1) has a positive solution.

Proof For $a=1000, b=1$, it is clear that $a>3 h_{0} h_{\tau}^{-1} b$. Moreover,

$$
f\left(t, x_{1}, x_{2}, x_{3}\right) \leq 2000 \leq h_{0}^{-1} a=2350
$$

for $\left(t, x_{1}, x_{2}, x_{3}\right) \in[0,1] \times[0,3000] \times[-3000,3000] \times[-3000,0]$, and

$$
f\left(t, x_{1}, x_{2}, x_{3}\right) \geq 2000\left(1-\frac{1}{1+\left(\frac{1}{4}\right)^{2}}\right) \geq 3 h_{\tau}^{-1} b
$$

for $\left(t, x_{1}, x_{2}, x_{3}\right) \in([0,1] \times[1 / 4,1] \times[-3,3] \times[-3,0]) \cup([0,1] \times[0,3] \times[-3,3] \times[-1,-1 / 4])$. Then BVP (4.1) has a positive solution by Theorem 3.2.

\section{Acknowledgements}

The authors express their sincere gratitude to the editors and anonymous referee for the careful reading of the original manuscript and thoughtful comments.

\section{Funding}

The project is supported by the Natural Science Foundation of China (61473065) and the National Training Program of Innovation and Entrepreneurship for Undergraduates (191034).

\section{Competing interests}

The authors declare that they have no competing interests.

\section{Authors' contributions}

GZ provided the idea of this article, all authors completed the paper, read and approved the final manuscript.

\section{Publisher's Note}

Springer Nature remains neutral with regard to jurisdictional claims in published maps and institutional affiliations.

Received: 18 June 2019 Accepted: 5 November 2019 Published online: 14 November 2019

\section{References}

1. Alves, E., Ma, T.F., Pelicer, M.L.: Monotone positive solutions for a fourth order equation with nonlinear boundary conditions. Nonlinear Anal. 71, 3834-3841 (2009)

2. Yao, Q.: Monotonically iterative method of nonlinear cantilever beam equations. Appl. Math. Comput. 205, 432-437 (2008)

3. Li, Y:: On the existence of positive solutions for the bending elastic beam equations. Appl. Math. Comput. 189, $821-827(2007)$

4. Ma, R.: Existence of positive solutions of a fourth-order boundary value problem. Appl. Math. Comput. 168 1219-1231 (2005)

5. Bai, Z.: Positive solutions of some nonlocal fourth-order boundary value problem. Appl. Math. Comput. 215, 4191-4197 (2010) 
6. Guo, Y., Yang, F., Liang, Y.: Positive solutions for nonlocal fourth-order boundary value problems with all order derivatives. Bound. Value Probl. (2012). https://doi.org/10.1186/1687-2770-2012-29

7. Li, Y: Existence of positive solutions for the cantilever beam equations with fully nonlinear terms. Nonlinear Anal., Real World Appl. 27, 221-237 (2016)

8. Kaufmann, E.R., Kosmatov, N.: Elastic beam problem with higher order derivatives. Nonlinear Anal., Real World Appl. 8, 811-821 (2007)

9. Li, Y.: Positive solutions of fourth-order boundary value problems with two parameters. J. Math. Anal. Appl. 281, 477-484 (2003)

10. Minhós, F., Gyulov, T., Santos, A.I.: Lower and upper solutions for a fully nonlinear beam equation. Nonlinear Anal. 71 , 281-292 (2009)

11. Yao, Q.: Local existence of multiple positive solutions to a singular cantilever beam equation. J. Math. Anal. Appl. 363 , 138-154 (2010)

12. Zhang, J., Zhang, G., Li, H.: Positive solutions of second-order problem with dependence on derivative in nonlinearity under Stieltjes integral boundary condition. Electron. J. Qual. Theory Differ. Equ. (2018). https://doi.org/10.14232/ejatde.2018.1.4

13. Ma, Y., Yin, C., Zhang, G.: Positive solutions of fourth-order problems with dependence on all derivatives in nonlinearity under Stieltjes integral boundary conditions. Bound. Value Probl. (2019). https://doi.org/10.1186/s13661-019-1155-7

14. Bai, Z., Du, Z., Zhang, S.: Iterative method for a class of fourth-order $p$-Laplacian beam equation. J. Appl. Anal. Comput. 9. 1443-1453 (2019)

15. Wei, Y., Song, Q., Bai, Z: Existence and iterative method for some fourth order nonlinear boundary value problems. Appl. Math. Lett. 87, 101-107 (2019)

16. Webb, J.R.L., Infante, G.: Non-local boundary value problems of arbitrary order. J. Lond. Math. Soc. 79, 238-259 (2009)

17. Infante, G., Pietramala, P.: A cantilever equation with nonlinear boundary conditions. Electron. J. Qual. Theory Differ. Equ. (2009). https://doi.org/10.14232/ejqtde.2009.4.15

18. Webb, J.R.L., Infante, G., Franco, D.: Positive solutions of nonlinear fourth-order boundary-value problems with local and non-local boundary conditions. Proc. R. Soc. Edinb. A 138, 427-446 (2008)

19. Deimling, K.: Nonlinear Functional Analysis. Springer, Berlin (1985)

20. Guo, D., Lakshmikantham, V:: Nonlinear Problems in Abstract Cones. Academic Press, San Diego (1988)

21. Avery, R., Henderson, J., O'Regan, D.: Functional compression-expansion fixed point theorem. Electron. J. Differ. Equ. 2008, Article ID 22 (2008)

\section{Submit your manuscript to a SpringerOpen ${ }^{\circ}$ journal and benefit from:}

- Convenient online submission

Rigorous peer review

- Open access: articles freely available online

- High visibility within the field

- Retaining the copyright to your article

Submit your next manuscript at $\gg$ springeropen.com 